\title{
LIPSCHITZ FUNCTIONS WITH UNEXPECTEDLY LARGE SETS OF NONDIFFERENTIABILITY POINTS
}

\author{
MARIANNA CSÖRNYEI, DAVID PREISS, AND JAROSLAV TIŠER
}

Received 12 January 2004

It is known that every $G_{\delta}$ subset $E$ of the plane containing a dense set of lines, even if it has measure zero, has the property that every real-valued Lipschitz function on $\mathbb{R}^{2}$ has a point of differentiability in $E$. Here we show that the set of points of differentiability of Lipschitz functions inside such sets may be surprisingly tiny: we construct a $G_{\delta}$ set $E \subset \mathbb{R}^{2}$ containing a dense set of lines for which there is a pair of real-valued Lipschitz functions on $\mathbb{R}^{2}$ having no common point of differentiability in $E$, and there is a realvalued Lipschitz function on $\mathbb{R}^{2}$ whose set of points of differentiability in $E$ is uniformly purely unrectifiable.

\section{Introduction and results}

One of the important results of Lebesgue tells us that Lipschitz functions on the real line are differentiable almost everywhere. This result is remarkably sharp: it is not difficult to see that for every Lebesgue null set $E$ on the real line there is a real-valued Lipschitz function which is nondifferentiable at any point of $E$. The higher-dimensional extension of Lebesgue's result, due to Rademacher, says that Lipschitz functions on $\mathbb{R}^{n}$ are also differentiable almost everywhere. Here, however, the sharpness of Lebesgue's theorem seems to be lost, as there are null sets in $\mathbb{R}^{2}$ in which every real-valued Lipschitz function has a point of differentiability. A plethora of such examples may be constructed using the following statement of [6], where it is proved not only in the plane, but in every Banach space with a smooth norm. Recall that a set is $G_{\delta}$ if it is an intersection of a sequence of open sets.

Theorem 1.1. Suppose that $E$ is a $G_{\delta}$ subset of $\mathbb{R}^{2}$ having the property that for any two points $u, v \in \mathbb{R}^{2}$ and for any $\varepsilon>0$ there is a Lipschitz $\gamma:[0,1] \mapsto \mathbb{R}^{2}$ such that $\|\gamma(0)-u\|<\varepsilon$, $\|\gamma(1)-v\|<\varepsilon, \int_{0}^{1}\left\|\gamma^{\prime}(t)-(v-u)\right\|<\varepsilon$, and $\mu\{t \in[0,1]: \gamma(t) \notin E\}<\varepsilon$. Then every realvalued Lipschitz function defined on a nonempty open subset of the plane is differentiable at some point of $E$.

The most well-known examples of sets $E$ satisfying the condition of Theorem 1.1 are constructed by requiring that the curves $\gamma$ be lines and that the Lebesgue measure 
$\mu\{t \in[0,1]: \gamma(t) \notin E\}$ be not only small, but the set is in fact empty. They are given by the formula

$$
E=\bigcap_{n=1}^{\infty} \bigcup_{k=n}^{\infty} B\left(L_{k}, \varrho_{k}\right)
$$

where $B(S, \varrho)$ denotes the set $\{z: \operatorname{dist}(z, S)<\varrho\}$ and $L_{k}$ is a sequence of lines in $\mathbb{R}^{2}$ which is dense in the space of lines; the latter condition means that for any $u, v \in \mathbb{R}^{2}$ and $\varepsilon>0$ there is $k$ such that both $u$ and $v$ are within distance $\varepsilon$ of $L_{k}$. The set $E$ has measure zero if $\sum_{k=1}^{\infty} \varrho_{k}<\infty$ and the set of lines contained in $E$ is always dense in the space of lines. This may be seen by noting that the sets $\left\{(u, v) \in \mathbb{R}^{2} \times \mathbb{R}^{2}: u \neq v,[u+n(u-v), v+n(v-u)] \subset\right.$ $\left.\bigcup_{k=n}^{\infty} B\left(L_{k}, \varrho_{k}\right)\right\}$ are open and dense in $\mathbb{R}^{4}$ and for any $(u, v)$ in their intersection (which is dense in $\mathbb{R}^{4}$ by the Baire category theorem) the line passing through $u, v$ lies in $E$.

Here we show that the set of points of differentiability of real-valued Lipschitz functions inside a particular set $E$ of the form described in (1.1), although nonempty by Theorem 1.1, may still be extremely small.

Our first example will give a pair of real-valued Lipschitz functions on $\mathbb{R}^{2}$ with no common points of differentiability in $E$; in other words, we construct a Lipschitz function $f: \mathbb{R}^{2} \rightarrow \mathbb{R}^{2}$ which is differentiable at no point of $E$. The example will even provide a function which is "uniformly nondifferentiable on $E$ " in the sense that the quantity

$$
\varepsilon^{*}(f, z)=\limsup _{r \rightarrow 0^{+}} \frac{\sup \{\|f(u)+f(v)-2 f((u+v) / 2)\|: u, v \in B(z, r)\}}{r}
$$

is, on $E$, bounded away from zero. In this connection, recall that the only known analogues of Theorem 1.1 for vector-valued functions do not show differentiability, but the so-called $\varepsilon$-differentiability. (See $[3,4]$ where the emphasis is on the infinite-dimensional case and [2] for a considerably more precise result in the finite-dimensional case. Here we ignore the results of [5] because they are purely infinite dimensional.) The concept of $\mathcal{\varepsilon}$-differentiability measures the nondifferentiability of $f: \mathbb{R}^{m} \rightarrow \mathbb{R}^{n}$ by the quantity

$$
\varepsilon(f, z)=\inf _{M} \limsup _{r \rightarrow 0^{+}} \frac{\sup \{\|f(u)-f(z)-M(u-z)\|: u \in B(z, r)\}}{r},
$$

where the infimum is over the set of $n \times m$ matrices. An $\varepsilon$-differentiability result for a set $E$ and a function $f$ would say that $E$ contain points with $\varepsilon(f, z)$ arbitrarily small; this is (considerably) stronger than requiring that the set $E$ contain points with $\varepsilon^{*}(f, z)$ arbitrarily small. Our example therefore shows that $\varepsilon$-differentiability results for vectorvalued functions cannot be extended to all sets for which we have full differentiability results for real-valued functions.

Our second example will provide a real-valued Lipschitz function on $\mathbb{R}^{2}$ whose set of differentiability points inside $E$ is small in the sense of rectifiability. Recall that a subset 
$N$ of $\mathbb{R}^{2}$ is called purely unrectifiable if it meets every rectifiable curve in a set of onedimensional measure zero. A somewhat stronger notion of uniform pure unrectifiability is defined by requiring the existence of an $\eta>0$ such that for every segment $I$ of the unit circle of length $\eta$ and for every $\varepsilon>0$ there is an open set $G$ containing $N$ with the property that $\mu\left(\gamma^{-1}(G)\right)<\varepsilon$ for every Lipschitz $\gamma:[0,1] \rightarrow \mathbb{R}^{2}$ such that $\gamma^{\prime}(t) \in I$ for almost every $t$. Although these are basic concepts, not much appears to be known about them. In particular, it is not known whether for $G_{\delta}$ sets the notions of pure and uniform pure unrectifiability coincide or not. Some information will eventually be found in [1]: an equivalent definition of uniform pure unrectifiability is obtained by fixing the $\eta$ as any number less than $\pi$, and for us the most relevant point is that uniform pure unrectifiability characterises the sets $N$ for which there is a real-valued Lipschitz function having no directional derivative at any point of $N$. Using this result, we could have easily obtained our first example from the second; we have not done it partly because the second example is considerably harder but mainly because in this way we would not obtain a uniform estimate of nondifferentiability of the pair of functions. We explain the reasoning behind this after stating our result.

THeOREM 1.2. There is a $G_{\delta}$ subset $E$ of $\mathbb{R}^{2}$ containing a dense set of lines for which we can construct

(i) a Lipschitz function $f: \mathbb{R}^{2} \rightarrow \mathbb{R}^{2}$ which is differentiable at no point of $E$, and which even satisfies that, for a fixed $\varepsilon>0, f$ is not $\varepsilon$-differentiable at any point of $E$,

(ii) a real-valued Lipschitz function on $\mathbb{R}^{2}$ whose set of points of differentiability in $E$ is uniformly purely unrectifiable.

As we have already pointed out, if we take the function, say $h$, from (ii) and use the result from [1] to find a real-valued Lipschitz function $g$ on $\mathbb{R}^{2}$ which is nondifferentiable at every point of the uniformly purely unrectifiable set $N$ of the points of differentiability of $h$ in $E$, the pair $(g, h)$ will provide an example satisfying the first part of (i). However, this would not easily provide an example of an $f: \mathbb{R}^{2} \rightarrow \mathbb{R}^{2}$ that is not $\varepsilon$-differentiable on $E$, since for every $\varepsilon>0$ the set of points $z \in E$ at which $\varepsilon(h, z)<\varepsilon$ must be of positive measure on some lines lying in $E$. (This is explained in [6] and is behind the $\varepsilon$-differentiability results alluded to above.) As we do not have any control of the behaviour of $g$ at most of these points, the proof of $\varepsilon$-nondifferentiability of $(g, h)$ would require further arguments.

Yet another curious difference between the one- and two-dimensional situation arises in this connection. To explain it, recall (a special case of) the result of Zahorski [7] that for every $G_{\delta}$ set $N \subset \mathbb{R}$ of measure zero there is $\psi: \mathbb{R} \rightarrow \mathbb{R}$ with $\operatorname{Lip}(\psi) \leq 1$, which is differentiable at every point of $\mathbb{R} \backslash N$, and at the points of $N$ it satisfies

$$
\limsup _{y \rightarrow x} \frac{\psi(y)-\psi(x)}{y-x}=1, \quad \liminf _{y \rightarrow x} \frac{\psi(y)-\psi(x)}{y-x}=-1
$$

This result may be used to show that the set of points of differentiability of a real-valued Lipschitz function $h$ that lie in a set $E$ satisfying the assumptions of Theorem 1.1 cannot be too small: its Hausdorff (one-dimensional) measure must be positive, since otherwise 
it would project to a null set on the $x$-axis and a suitable linear combination of $h$ and Zahorski's function $\psi$ would provide a Lipschitz function differentiable at no points of $E$. (A stronger version of Zahorski's results is used in [6] to show that the one-dimensional projections of the set of points of differentiability of a real-valued Lipschitz function that lie in a set $E$ satisfying the assumptions of Theorem 1.1 have a null complement.) Now, a seemingly plausible version of Zahorski's result in the plane may say that for every uniformly purely unrectifiable $G_{\delta}$ set $N \subset \mathbb{R}^{2}$ there is a Lipschitz $\psi: \mathbb{R}^{2} \rightarrow \mathbb{R}$ that is differentiable at every point of $\mathbb{R}^{2} \backslash N$ and satisfies $\varepsilon(\psi, z) \geq \varepsilon>0$, for all $z \in N$. But this is false whenever $N$ contains the set of points of the set $E$ from Theorem 1.2 at which the function $h$ from (ii) is differentiable, because then a suitable linear combination of $h$ and $\psi$ would be differentiable at no points of $E$. Notice that there are such uniformly purely unrectifiable $G_{\delta}$ sets $N$ since every uniformly purely unrectifiable set is obviously contained in a uniformly purely unrectifiable $G_{\delta}$ set.

\section{Constructions}

We first describe the method of the choice of the lines $L_{1}, L_{2}, \ldots$ and the half-widths $\varrho_{k}>0$ of the strips $B\left(L_{k}, \varrho_{k}\right)$ which is common to both examples. In addition to $L_{k}$ and $\varrho_{k}$, we will also construct functions $g_{k}: \mathbb{R}^{2} \rightarrow \mathbb{R}^{2}$ in the first example or $\varphi_{k}: \mathbb{R}^{2} \rightarrow \mathbb{R}$ in the second example, and a finite set of lines which we wish to avoid in the future choices of lines; we denote by $T_{k}$ the union of these "prohibited" lines. The function $f$ for the first example will be obtained as a composition of the $g_{k}$, and the function $h$ for the second example as a sum of multiples of the $\varphi_{k}$ by suitable functions.

The recursive construction will run as follows. We order a countable dense subset of $\mathbb{R}^{4}$ into a sequence $\left(u_{k}, v_{k}\right)$ and start the induction by choosing $L_{0}$ and $\varrho_{0}$ arbitrarily and letting $T_{0}=\partial B\left(L_{0}, \varrho_{0}\right)$. Whenever $L_{j}, \varrho_{j}, g_{j}$ or $\varphi_{j}$, and $T_{j}$ have been defined for $j<k$, we choose a line $L_{k}$ not lying in $T_{k-1}$ which passes within $1 / k$ of both $u_{k}$ and $v_{k}$ (and satisfying another simple condition in the first example). Then we define $\varrho_{k}$ by requirements that make it small compared to the data we have so far and continue by defining the functions $g_{k}$ or $\varphi_{k}$. These functions will be piecewise affine, and we choose a finite union of lines $T_{k} \supset T_{k-1} \cup \partial B\left(L_{k}, \varrho_{k}\right)$ so that they are affine on every component of $\mathbb{R}^{2} \backslash T_{k}$; in the first example, we also require that several other functions obtained by composition of $g_{j}, j \leq$ $k$, be affine on every component of $\mathbb{R}^{2} \backslash T_{k}$. Although the particular requirements on the various choices will be somewhat different in the two constructions; it is clear that we can satisfy both of them at the same time and so get the same set $E$ (which is, of course, defined by (1.1)).

The notation we use is either mostly standard or easy to understand, such as $\langle u, v\rangle$ for the scalar product of the vectors $u$ and $v$. On two occasions, we find it convenient to use the less standard notation for the cutoff function, which is defined by cutoff $(x, y)=$ $\min (\max (x,-y), y)$ for $x \in \mathbb{R}$ and $y \geq 0$.

2.1. Proof of Theorem 1.2(i). For this example, we additionally require that the line $L_{k}$ do not pass through any meeting point of two different lines of $T_{k-1}$, and that it is not perpendicular to any line of $T_{k-1}$. The choice of $\varrho_{k}$ is subject to the conditions that $\varrho_{k} \leq \varrho_{k-1} / 12$ and that, for any $z \in L_{k}, B\left(z, \varrho_{k}\right)$ meets no more than one of the lines of 
which $T_{k-1}$ consists. The function $g_{k}: \mathbb{R}^{2} \rightarrow \mathbb{R}^{2}$ will be defined by

$$
g_{k}(z)=z-2 \operatorname{cutoff}\left(\left\langle z, v_{k}\right\rangle-\alpha_{k}, \varrho_{k}\right) v_{k}
$$

where $v_{k}$ is a unit vector perpendicular to $L_{k}$ and $\alpha_{k}=\left\langle u, v_{k}\right\rangle$ for $u \in L_{k}$. Geometrically, this definition says that, in the strip $B\left(L_{k}, \varrho_{k}\right), g_{k}$ is the reflection about $L_{k}$, and each of the remaining half-planes is shifted perpendicularly to $L_{k}$ so that each of the two lines forming the boundary $\partial B\left(L_{k}, \varrho_{k}\right)$ of the strip is mapped onto the other one. Finally, $T_{k} \supset$ $T_{k-1} \cup \partial B\left(L_{k}, \varrho_{k}\right)$ is chosen so that all compositions $g_{j} \circ g_{j+1} \circ \cdots \circ g_{k}$, where $j \leq k$, are affine on every component of $\mathbb{R}^{2} \backslash T_{k}$.

For $j \leq k$, we let

$$
f_{j, k}=g_{j} \circ g_{j+1} \circ \cdots \circ g_{k-1}
$$

with the usual convention that the composition of an empty sequence of functions is the identity. Noting that $g_{k}$ is an (affine) isometry on each of the three regions into which the plane is divided by $\partial B\left(L_{k}, \varrho_{k}\right)$, we see that $f_{j, k+1}$ is an affine isometry on each component of $\mathbb{R}^{2} \backslash T_{k}$.

Since $\left\|g_{j}(z)-z\right\| \leq 2 \varrho_{j}$ for every $z \in \mathbb{R}^{2}$, we have, for $j \leq k \leq l$ and $u \in \mathbb{R}^{2}$,

$$
\begin{gathered}
\left\|f_{k, l}(u)-u\right\| \leq \sum_{i=k}^{l-1}\left\|g_{i}\left(f_{i+1, l}(u)\right)-f_{i+1, l}(u)\right\| \leq \sum_{i=k}^{l-1} 2 \varrho_{i} \leq 3 \varrho_{k}, \\
\left\|f_{j, k}(u)-f_{j, l}(u)\right\| \leq\left\|f_{k, l}(u)-u\right\| \leq 3 \varrho_{k} .
\end{gathered}
$$

So the limits

$$
f_{j}=\lim _{k \rightarrow \infty} f_{j, k}
$$

exist and, $\operatorname{since} \operatorname{Lip}\left(g_{i}\right) \leq 1$ for each $i$, we have $\operatorname{Lip}\left(f_{j}\right) \leq 1$. Moreover, for each $j \leq k$,

$$
f_{j}=f_{j, k} \circ f_{k}=f_{j, k} \circ g_{k} \circ f_{k+1} .
$$

We show that $f=f_{1}$ is the required function. For this, assume that $z \in E$ and consider any $k$ such that $z \in B\left(L_{k}, \varrho_{k}\right)$. Let $u \in L_{k}$ and $v_{1}, v_{2} \in \partial B\left(L_{k}, \varrho_{k}\right), v_{1} \neq v_{2}$, lie on the line through $z$ perpendicular to $L_{k}$. By the choice of $\varrho_{k}$, $\left[v_{1}, v_{2}\right]$ may meet at most one line of $T_{k-1}$, hence the interior of one of the segments $\left[u, v_{1}\right],\left[u, v_{2}\right]$ does not cross any line of $T_{k-1}$. Choose the notation so that it is $\left[u, v_{1}\right]$ and define $v=u+2\left(v_{2}-u\right)$. Then $f_{1, k}$ is an affine isometry on $g_{k}([u, v])=\left[u, v_{1}\right]$ and hence by (2.3) and (2.5),

$$
\begin{aligned}
\| f(u) & +f(v)-2 f\left(\frac{(u+v)}{2}\right) \| \\
\geq & \left\|f_{1, k}\left(g_{k}(u)\right)+f_{1, k}\left(g_{k}(v)\right)-2 f_{1, k}\left(g_{k}\left(\frac{(u+v)}{2}\right)\right)\right\|-12 \varrho_{k+1} \\
= & \left\|g_{k}(u)+g_{k}(v)-2 g_{k}\left(\frac{(u+v)}{2}\right)\right\|-12 \varrho_{k+1} \\
& =2 \varrho_{k}-12 \varrho_{k+1} \geq \varrho_{k} .
\end{aligned}
$$


Since the distance of the points $u, v$ from $z$ is not more than $3 \varrho_{k}$, this means that $\varepsilon^{*}(f, z) \geq$ $1 / 3$.

2.2. Proof of Theorem 1.2(ii). Here we do not need any further conditions on the choice of $L_{k}, k \geq 1$. Before choosing $\varrho_{k}$, we let $S_{k}=L_{k} \cap T_{k-1}$, denote by $s_{k}$ the number of elements of $S_{k}$ and choose $0<\delta_{k}<2^{-k-3} / s_{k}$. We also choose a unit vector $e_{k}$ parallel to $L_{k}$ and denote $\alpha_{k}=\left\langle z, e_{k}^{\perp}\right\rangle$ where $z \in L_{k}$; we use the notation $u^{\perp}=\left(-u_{2}, u_{1}\right)$ for $u=$ $\left(u_{1}, u_{2}\right)$. We subject $\varrho_{k}$ to the conditions $\varrho_{k}<16^{-k-3} \sin (\pi / 36), \varrho_{k} \leq \varrho_{k-1} / 32$, and $\varrho_{k}<$ $2^{-k-1} \operatorname{dist}\left(z, T_{k-1}\right)$ for $z \in B\left(L_{k}, \varrho_{k}\right) \backslash B\left(S_{k}, \delta_{k}\right)$. The last assumption implies

$$
B\left(z, 4 \varrho_{k}\right) \cap T_{k-1}=\varnothing \quad \text { for } z \in B\left(L_{k}, \varrho_{k}\right) \backslash B\left(S_{k}, \delta_{k}\right) .
$$

Finally, we define $T_{k} \supset T_{k-1} \cup \partial B\left(L_{k}, \varrho_{k}\right)$ so that the function

$$
\varphi_{k}(z)=\operatorname{cutoff}\left(\left\langle z, e_{k}^{\perp}\right\rangle-\alpha_{k}, \min \left(\varrho_{k}, 2^{-k} \operatorname{dist}\left(z, T_{k-1}\right)\right)\right)
$$

is affine on each component of $\mathbb{R}^{2} \backslash T_{k}$.

We let

$$
C_{k}=\sum_{j=0}^{k-1} 2^{-j}(4 j+24)
$$

these constants will be used to control the Lipschitz constant of a sequence of functions approximating the desired function $h$. We list here the inequalities involving $\delta_{k}$ and $\varrho_{k}$ in a form that will be actually used:

$$
\sum_{j=k}^{\infty}\left(3 \delta_{j} s_{j}+2 \varrho_{j} \csc \left(\frac{\pi}{36}\right)\right)<2^{-k}, \quad \sum_{j=k+1}^{\infty} 4 \varrho_{j}<\frac{\varrho_{k}}{4}, \quad \sum_{j=k}^{\infty} 3^{j-1} 6 \varrho_{j}<4^{-k}
$$

We start our construction by defining four sequences of functions that describe various aspects of the geometry of the strips $B\left(L_{k}, \varrho_{k}\right)$. Each of them will have the property that the $k$ th function is constant on each component of $\mathbb{R}^{2} \backslash \bigcup_{j=1}^{k} \partial B\left(L_{j}, \varrho_{j}\right)$.

(1) Let $k_{0}(z)=0$ and $k_{p}(z)=\min \left\{k>k_{p-1}(z): z \in B\left(L_{k}, \varrho_{k}\right)\right\}$; this formula is understood to imply that $k_{p}(z)=\infty$ if $z \notin \bigcup_{k>k_{p-1}(z)} B\left(L_{k}, \varrho_{k}\right)$.

(2) Put $\sigma_{j}(z)=(-1)^{p}$ if $k_{p}(z) \leq j<k_{p+1}(z)$.

(3) Choose $W \subset\left\{z \in \mathbb{R}^{2}:\|z\|=1\right\}$ having five elements so that for every line $L$ there is $w \in W$ whose angle with $L$ is no more than $\pi / 9$. We also pick $w_{0} \in W$ and let $w_{0}(z)=w_{0}$. If $U$ is a component of $B\left(L_{k}, \varrho_{k}\right) \backslash \bigcup_{j=1}^{k-1} \partial B\left(L_{j}, \varrho_{j}\right)$ on which the angle between $w_{k-1}(z)$ and $L_{k}$ is bigger than $2 \pi / 9$ (notice that this angle does not depend on $z \in U$, since $w_{k-1}$ is constant on $U$ ), then we choose $w \in W$ whose angle with $L_{k}$ is no more than $\pi / 9$ and let $w_{k}(z)=w$ for $z \in U$. In all other cases, we let $w_{k}(z)=w_{k-1}(z)$.

(4) Put $\zeta_{k}(z)=1 /\left\langle e_{k+1}, w_{k}(z)\right\rangle$ if $\left|\left\langle e_{k+1}, w_{k}(z)\right\rangle\right| \geq 1 / 2$ and $\zeta_{k}(z)=0$ otherwise. 
The functions $h_{k}$ approximating $h$ will be defined as a combination of the functions $\varphi_{k}$ defined in (2.8). Notice that $\varphi_{k}$ is continuous on $\mathbb{R}^{2}$, affine on each component of $\mathbb{R}^{2} \backslash T_{k},\left|\varphi_{k}(z)\right| \leq \varrho_{k},\left\|\varphi_{k}^{\prime}(z)\right\| \leq 1$, and $\left\|\varphi_{k}^{\prime}(z)\right\| \leq 2^{-k}$ for $z \notin B\left(L_{k}, \varrho_{k}\right)$. Note also that $\varphi_{k}$ is zero on $T_{k-1}$, on the components of the complement of which both $\sigma_{k-1}$, and $\zeta_{k-1}$ are constant.

The coefficients of the required combination of the $\varphi_{k}$ will depend on yet another sequence $m_{k}$ of integer-valued functions on $\mathbb{R}^{2}$; these functions will be constant on the components of $\mathbb{R}^{2} \backslash T_{k}$ and, similarly to the $\varphi_{k}$, the functions $h_{k}$ approximating $h$ will be continuous on $\mathbb{R}^{2}$ and affine on each such component. These functions are defined by requiring that

(i) $m_{0}(z)=0$ and $h_{0}(z)=0$ for all $z \in \mathbb{R}^{2}$;

(ii) $h_{k}(z)=h_{k-1}(z)+2^{-m_{k-1}(z)} \sigma_{k-1}(z) \zeta_{k-1}(z) \varphi_{k}(z)$;

(iii) $m_{k}(z)=m_{k-1}(z)+1$ if $z \notin T_{k}$ and $\left\|h_{k}^{\prime}(z)\right\|>C_{m_{k-1}(z)}$;

(iv) $m_{k}(z)=m_{k-1}(z)$ in all other cases.

The function with a small set of points of differentiability is defined by

$$
h(z)=\sum_{k=1}^{\infty} 2^{-m_{k-1}(z)} \sigma_{k-1}(z) \zeta_{k-1}(z) \varphi_{k}(z)=\lim _{k \rightarrow \infty} h_{k}(z)
$$

the series converges since $\left|\zeta_{k-1}(z)\right| \leq 2$ and so its terms are bounded by $2 \varrho_{k}$, where $\sum_{k} \varrho_{k}$ converges.

Notice that $m_{k-1}$ is constant on each component of $\mathbb{R}^{2} \backslash T_{k-1}$ and that $\varphi_{k}$ is zero on $T_{k-1}$, so $h_{k}$ is continuous on $\mathbb{R}^{2}$ and affine on each component of $\mathbb{R}^{2} \backslash T_{k}$. In particular, the functions $h_{k}$ are Lipschitz. To show that $h$ is Lipschitz as well, we show that

$$
\left\|h_{k}^{\prime}(z)\right\| \leq C_{m_{k}} \quad \text { for every } z \notin T_{k} .
$$

This clearly holds for $k=0$ and, if it holds for $k-1$, then either $\left\|h_{k}^{\prime}(z)\right\| \leq C_{m_{k-1}} \leq C_{m_{k}}$ or $m_{k}$ was defined in (iii), so $m_{k}=m_{k-1}+1$ and $\left\|h_{k}^{\prime}(z)\right\| \leq C_{m_{k-1}}+2^{-m_{k-1}+1} \leq C_{m_{k-1}+1}=C_{m_{k}}$.

Since the sequence $C_{j}$ is bounded, (2.12) implies that the Lipschitz constants of $h_{k}$ are bounded by a constant independent of $k$ and hence $h$ is Lipschitz.

We need to show that the set of the points of differentiability of $h$ in $E$ is uniformly purely unrectifiable. We choose $\eta=\pi / 18$ in the definition of uniform pure unrectifiability, and let $I$ be an arc of the unit circle of length $\pi / 18$. Denote by $I_{1}$ and $I_{2}$ the arcs of the unit circle concentric with $I$ of length $\pi / 9$ and $5 \pi / 9$, respectively. These angles fit with the definition of $w_{k}$ : they are chosen so that the angle between any vector $e \in I_{1}$ and $w \in I_{2}$ is no more than $\pi / 3$ and if the angle between some $e \in I_{1}$ and $w$ does not exceed $\pi / 9$, then $w \in I_{2}$ and the angle between $w$ and any $e \in I_{1}$ does not exceed $2 \pi / 9$.

For $n=1,2, \ldots$, denote

$$
\begin{aligned}
G_{n} & =\bigcup_{k \geq n, \pm e_{k} \notin I_{1}} B\left(L_{k}, \varrho_{k}\right) \cup \bigcup_{k \geq n} B\left(S_{k}, \delta_{k}\right), \\
H_{n} & =\left\{z: \sup _{k} m_{k}(z)>n+1\right\} .
\end{aligned}
$$


These sets are open: for $G_{n}$ this is obvious and for $H_{n}$ it follows by observing that the functions $m_{k}$ are lower semicontinuous. It is our intention to show that the sets $G_{n} \cup H_{n}$ form the required open covers of the set of points of differentiability of $h$ in $E$. For this purpose, we fix $n$ and start with proving the following statement.

Claim 2.1. Let $z \in \mathbb{R}^{2} \backslash G_{n}$ and simplify the notation by writing $k_{p}$ for $k_{p}(z)$ and $w_{k}$ for $w_{k}(z)$. Then for any $p$ such that $k_{p} \geq n$,

(i) $e_{k_{q}} \in \pm I_{1}$ for $q \geq p$,

(ii) $\varphi_{k_{q}}(z)=\left\langle z, e_{k_{q}}^{\perp}\right\rangle-\alpha_{k_{q}}$ for $q \geq p$,

(iii) $w_{k} \in \pm I_{2}$ for all $k \geq k_{p}$,

and there is $r \geq p$ such that

(iv) $w_{k}=w_{k_{p}}$ for $k_{p} \leq k<k_{r}$, and $w_{k}=w_{k_{r}}$ for $k \geq k_{r}$,

(v) $\zeta_{k_{q}-1}(z)=1 /\left\langle e_{k_{q}}, w_{k_{p}}\right\rangle$ for $p<q<r$, and $\zeta_{k_{q}-1}(z)=1 /\left\langle e_{k_{q}}, w_{k_{r}}\right\rangle$ for $q>r$.

The statement (i) follows immediately from $z \in B\left(L_{k_{q}}, \varrho_{k_{q}}\right)$ and $z \notin G_{n}$, and the statement (ii) follows from $z \in B\left(L_{k_{q}}, \varrho_{k_{q}}\right) \backslash B\left(S_{k_{q}}, \delta_{k_{q}}\right)$ since for such $z$ we have $\varrho_{k_{q}}<2^{-k_{q}} \operatorname{dist}(z$, $\left.T_{k_{q}-1}\right)$. For the remaining statements, first notice that $w_{k}$ stays constant for $k_{q-1} \leq k<k_{q}$ and that the angle between $w_{k_{q}}$ and $L_{k_{q}}$ never exceeds $2 \pi / 9$. Hence, by (i) and the definition of $I_{2}, w_{k_{q}} \in \pm I_{2}$ for $q \geq p$, and so $w_{k} \in \pm I_{2}$ for all $k \geq k_{p}$ as claimed in (iii). The statement (iv) is obvious by letting $r=p$ if $w_{k}=w_{k_{p}}$ for all $k \geq k_{p}$. If this is not the case, take the least index after $k_{p}$, which must necessarily be of the form $k_{r}$, for which $w_{k_{r}} \neq w_{k_{p}}$. Then $w_{k}=w_{k_{p}}$ for $k_{p} \leq k<k_{r}$, and the definition of $w_{k_{r}}$ gives that the angle between $w_{k_{r}}$ and $L_{k_{r}}$ does not exceed $\pi / 9$. Since by (i) $e_{k_{q}} \in \pm I_{1}$, the angle between $w_{k_{r}}$ and any $e_{k_{q}}$, $q \geq r$, never exceeds $2 \pi / 9$. Hence, $w_{k_{q}}=w_{k_{r}}$ for $q \geq r$ and (iv) follows. From (i) and (iii), we infer that the angle between $e_{k_{q}}$ and $w_{k_{q}-1}=w_{k_{p}}$ did not exceed $\pi / 3$, and (v) follows from (iv).

We now show that $h$ is nondifferentiable at any point $z \in E \backslash\left(G_{n} \cup H_{n}\right)$. Indeed, since $z \in E, k_{p}(z)<\infty$ for all $p$. So, since $z \notin H_{n}$, there is an index $p$ such that $k_{p} \geq n$ and $m:=m_{k_{p}}(z)=m_{j}(z)$ for all $j \geq k_{p}$. By Claim 2.1, $w_{k}(z) \in \pm I_{2}$ for all $k \geq k_{p}(z)$, and $e_{k_{q}(z)} \in \pm I_{1}$ for $q \geq p$. Consider any $q>p$ and denote $k=k_{q}(z)$. Since the angle between $w_{k-1}(z)$ and $L_{k}$ does not exceed $\pi / 3,\left|\zeta_{k-1}(z)\right| \geq 1$ and there are $u \in L_{k}$ and $v \in$ $\partial B\left(L_{k}, 2 \varrho_{k}\right)$ so that $v-u$ is a multiple of $w_{k-1}(z)^{\perp}$ and $z$ lies on the line segment $[u, v]$; moreover, $\|v-u\| \leq 4 \varrho_{k}$. So, deducing from (2.7) that $h_{k-1}$ is affine on $B\left(z, 4 \varrho_{k}\right)$ and that $\varphi_{k}(u)=0$ and $\varphi_{k}(v)=\varphi_{k}((u+v) / 2)$ and they are either both $\varrho_{k}$ or both $-\varrho_{k}$, we use that $\sum_{j=k+1}^{\infty}\left|\varphi_{j}(u)+\varphi_{j}(v)-2 \varphi_{j}((u+v) / 2)\right| \leq \sum_{j=k+1}^{\infty} 4 \varrho_{j} \leq \varrho_{k} / 4$ to estimate $\mid h(u)+$ $h(v)-2 h((u+v) / 2) \mid \geq 2^{-m}\left(\left|\varphi_{k}(u)+\varphi_{k}(v)-2 \varphi_{k}((u+v) / 2)\right|-\varrho_{k} / 2\right)=2^{-m-1} \varrho_{k}$, which means that $\varepsilon^{*}(h, z) \geq 2^{-m-3}>0$.

It follows that the proof will be finished once we find $\varepsilon_{n} \rightarrow 0$ (independent of $\gamma$ ) so that $\mu\left(\gamma^{-1}\left(G_{n} \cup H_{n}\right)\right) \leq \varepsilon_{n}$. Since $G_{n} \cup H_{n}$ is open, it suffices to verify this inequality for a dense set of $\gamma$ (in the topology of uniform convergence), so we may and will assume that $\gamma$ intersects each $T_{k}$ in at most finitely many points and so all $h_{j}$ are differentiable at $\gamma(t)$, for almost every $t \in[0,1]$.

The estimate of the measure of $\gamma^{-1}\left(G_{n}\right)$ is straightforward. Since $I$ has length $\pi / 18$, and $2 \delta \sec (\pi / 36)<2 \delta \sec (\pi / 4)<3 \delta$, the $\gamma$-preimage of any disk of radius $\delta$ is contained in an interval of length at most $3 \delta$ and, if $e_{k} \notin \pm I_{1}$, the $\gamma$-preimage of $B\left(L_{k}, \varrho_{k}\right)$ is contained in 
an interval of length at most $2 \varrho_{k} \csc (\pi / 36)$. Hence,

$$
\begin{aligned}
\mu\left(\gamma^{-1}\left(G_{n}\right)\right) & \leq \sum_{k \geq n}^{\infty} \sum_{z \in S_{k}} \mu\left(\gamma^{-1}\left(B\left(z, \delta_{k}\right)\right)\right)+\sum_{k \geq n, e_{k} \notin \pm I_{1}}^{\infty} \mu\left(\gamma^{-1}\left(B\left(L_{k}, \varrho_{k}\right)\right)\right) \\
& \leq \sum_{k=n}^{\infty}\left(3 \delta_{k} s_{k}+2 \varrho_{k} \csc \left(\frac{\pi}{36}\right)\right)<2^{-n} .
\end{aligned}
$$

To estimate $\mu\left(\gamma^{-1}\left(H_{n} \backslash G_{n}\right)\right)$, we have to work a little bit more. Let $\Sigma_{p}$ be the least $\sigma$ algebra of subsets of $[0,1]$ with respect to which the functions $k_{q} \circ \gamma, 0 \leq q \leq p$ are measurable. Then the conditional expectations $\beta_{p}=\mathbb{E}\left(\gamma^{\prime} \mid \Sigma_{p}\right)$ form an $\mathbb{R}^{2}$-valued martingale such that $\left\|\beta_{p}\right\|_{\infty} \leq 1$.

For any $k$, the set $B\left(L_{k}, \varrho_{k}\right) \backslash \bigcup_{j<k} \partial B\left(L_{j}, \varrho_{j}\right)$ has at most $3^{k-1}$ components. Let $P$ denote one of these components. Then there is an index $p$ so that $k=k_{p}(z)$ for all $z \in P$. We show that

$$
\int_{\gamma^{-1}(P)}\left|\left\langle\beta_{p}(t), e_{k}^{\perp}\right\rangle\right| d t=\left|\int_{\gamma^{-1}(P)}\left\langle\gamma^{\prime}(t), e_{k}^{\perp}\right\rangle d t\right| \leq 6 \varrho_{k} .
$$

Since all $k_{q} \circ \gamma, 0 \leq q \leq p$ are constant on $\gamma^{-1}(P)$, so is $\beta_{p}$. Hence,

$$
\int_{\gamma^{-1}(P)}\left|\left\langle\beta_{p}(t), e_{k}^{\perp}\right\rangle\right| d t=\left|\int_{\gamma^{-1}(P)}\left\langle\beta_{p}(t), e_{k}^{\perp}\right\rangle d t\right|,
$$

and the equality follows from the definition of conditional expectations. The inequality is obvious if $P$ does not meet $\gamma$ or if the angle between $L_{k}$ and all vectors from $I$ is at least $\pi / 6$, since then $\gamma^{-1}(P)$ is contained in an interval of length at most $4 \varrho_{k}$. When the angle between $L_{k}$ and some vector from $I$ is less than $\pi / 6$, the function $t \rightarrow\left\langle\gamma(t), e_{k}\right\rangle$ is strictly monotonic. Let $a=\inf \left\{\left\langle z, e_{k}\right\rangle: z \in P\right\}$ and $b=\sup \left\{\left\langle z, e_{k}\right\rangle: z \in P\right\}$. Since $P$ is an open convex set, there are functions $\psi^{-}$and $\psi^{+}$on $(a, b)$ such that $\psi^{-}$is convex, $\psi^{+}$is concave, $\psi^{-}<\psi^{+}$, and $\partial P \cap\left\{z: a<\left\langle z, e_{k}\right\rangle<b\right\}$ is the union of the graphs of $\psi^{-}$and $\psi^{+}$(in the coordinate system $\left.e_{k}, e_{k}^{\perp}\right)$. By our assumption on $\gamma, \partial P$ meets $\gamma$ only in a finite set, hence $\gamma^{-1}(P)$ is the union of finitely many intervals, say $\left(a_{1}, a_{2}\right),\left(a_{3}, a_{4}\right), \ldots,\left(a_{2 d-1}, a_{2 d}\right)$, where $\left\langle\gamma\left(a_{1}\right), e_{k}\right\rangle,\left\langle\gamma\left(a_{2}\right), e_{k}\right\rangle, \ldots$ is strictly monotonic and for each $1 \leq i \leq d-1$ both points $\gamma\left(a_{2 i}\right)$ and $\gamma\left(a_{2 i+1}\right)$ lie either on the graph of $\psi^{-}$or on the graph of $\psi^{+}$. Since $\psi^{-}$is convex and oscillates between $\alpha_{k}-\varrho_{k}$ and $\alpha_{k}+\varrho_{k}$, the sum of $\left\langle\gamma\left(a_{2 i+1}\right)-\gamma\left(a_{2 i}\right), e_{k}^{\perp}\right\rangle=$ $\psi^{-}\left(\left\langle\gamma\left(a_{2 i+1}\right), e_{k}\right\rangle\right)-\psi^{-}\left(\left\langle\gamma\left(a_{2 i}\right), e_{k}\right\rangle\right)$ over those $i$ for which the first case occurs is at most $2 \varrho_{k}$. Similarly, we obtain the same estimate of the sum of $\left\langle\gamma\left(a_{2 i+1}\right)-\gamma\left(a_{2 i}\right), e_{k}^{\perp}\right\rangle$ over those $i$ for which the second case occurs. Hence,

$$
\begin{aligned}
& \left|\sum_{i=1}^{d}\left\langle\gamma\left(a_{2 i}\right)-\gamma\left(a_{2 i-1}\right), e_{k}^{\perp}\right\rangle\right| \\
& \quad \leq\left|\left\langle\gamma\left(a_{2 d}\right)-\gamma\left(a_{1}\right), e_{k}^{\perp}\right\rangle\right|+\left|\sum_{i=1}^{d-1}\left\langle\gamma\left(a_{2 i+1}\right)-\gamma\left(a_{2 i}\right), e_{k}^{\perp}\right\rangle\right| \leq 6 \varrho_{k},
\end{aligned}
$$

and (2.15) is proved. 
For any fixed $p$, by summing (2.15) first over those components $P$ of $B\left(L_{k}, \varrho_{k}\right) \backslash$ $\bigcup_{j<k} \partial B\left(L_{j}, \varrho_{j}\right)$ for which $k_{p}(z)=k$ on $P$, which gives no more than $3^{k-1}$ terms, and then over $k$, which starts only from $p$, we get that

$$
\int_{A_{p}}\left|\left\langle\beta_{p}(t), e_{k_{p}(\gamma(t))}^{\perp}\right\rangle\right| d t \leq \sum_{k=p}^{\infty} 3^{k-1} 6 \varrho_{k}<4^{-p},
$$

where $A_{p}=\left\{t: k_{p}(\gamma(t))<\infty\right\}$.

Hence, letting

$$
D_{p}:=\left\{t: k_{p}(\gamma(t))<\infty \text { and }\left|\left\langle\beta_{p}(t), e_{k_{p}(\gamma(t))}^{\perp}\right\rangle\right|>2^{-p}\right\},
$$

we conclude from the Markov inequality that

$$
\mu\left(D_{p}\right)<2^{-p}
$$

For each $v \in I_{2}$, we infer from $\gamma^{\prime}(t) \in I \subset I_{1}$ that $1 / 2 \leq\left\langle\gamma^{\prime}(t), v\right\rangle \leq 1$. Hence,

$$
\mu^{v}(A):=\frac{\int_{A}\left\langle\gamma^{\prime}, v\right\rangle d t}{\int_{0}^{1}\left\langle\gamma^{\prime}, v\right\rangle d t}
$$

is a well-defined probability measure on $[0,1]$. Since $\mathbb{E}\left(\left\langle\gamma^{\prime}, v\right\rangle \mid \Sigma_{p}\right)=\left\langle\beta_{p}, v\right\rangle$ and $\mathbb{E}\left(\left\langle\gamma^{\prime}\right.\right.$, $\left.\left.v^{\perp}\right\rangle \mid \Sigma_{p}\right)=\left\langle\beta_{p}, v^{\perp}\right\rangle$,

$$
\begin{aligned}
\mathbb{E}\left(\frac{\left\langle\beta_{p}, v^{\perp}\right\rangle}{\left\langle\beta_{p}, v\right\rangle} \cdot\left\langle\gamma^{\prime}, v\right\rangle \mid \Sigma_{p}\right) & =\frac{\left\langle\beta_{p}, v^{\perp}\right\rangle \cdot \mathbb{E}\left(\left\langle\gamma^{\prime}, v\right\rangle \mid \Sigma_{p}\right)}{\left\langle\beta_{p}, v\right\rangle}=\left\langle\beta_{p}, v^{\perp}\right\rangle \\
& =\mathbb{E}\left(\left\langle\gamma^{\prime}, v^{\perp}\right\rangle \mid \Sigma_{p}\right)=\mathbb{E}\left(\frac{\left\langle\gamma^{\prime}, v^{\perp}\right\rangle}{\left\langle\gamma^{\prime}, v\right\rangle} \cdot\left\langle\gamma^{\prime}, v\right\rangle \mid \Sigma_{p}\right) .
\end{aligned}
$$

Therefore, $\left\langle\beta_{p}, v^{\perp}\right\rangle /\left\langle\beta_{p}, v\right\rangle$ is a real-valued martingale with respect to the measure $\mu^{v}$ and filtration $\Sigma_{p}$. Since both $\left\langle\beta_{p}, v^{\perp}\right\rangle$ and $\left\langle\beta_{p}, v\right\rangle$ are in the interval $[1 / 2,1]$, the martingale is bounded by 2 . From this, it follows that the $L^{2}\left(\mu^{v}\right)$ norm of the martingale is bounded by 2 , moreover,

$$
\left\|\frac{\left\langle\beta_{0}, v^{\perp}\right\rangle}{\left\langle\beta_{0}, v\right\rangle}\right\|_{L^{2}\left(\mu^{v}\right)}^{2}+\sum_{p=1}^{\infty}\left\|-\frac{\left\langle\beta_{2 p-1}, v^{\perp}\right\rangle}{\left\langle\beta_{2 p-1}, v\right\rangle}+\frac{\left\langle\beta_{2 p}, v^{\perp}\right\rangle}{\left\langle\beta_{2 p}, v\right\rangle}\right\|_{L^{2}\left(\mu^{v}\right)}^{2} \leq 4 .
$$

Let

$$
\beta_{p}^{v}=\sum_{q=0}^{p}(-1)^{q} \frac{\left\langle\beta_{q}, v^{\perp}\right\rangle}{\left\langle\beta_{q}, v\right\rangle}
$$

Then $\beta_{2 p-1}^{v}$ is a $\mu^{v}$ martingale with respect to the $\sigma$-algebras $\Sigma_{2 p-1}$ with $L^{2}\left(\mu^{v}\right)$-norm bounded by 2 . By Kolmogorov's martingale inequality, $\mu^{v}\left\{t: \sup _{p}\left|\beta_{2 p-1}^{v}\right|>n\right\}<4 / n^{2}$. Since the terms of the series defining $\beta_{p}^{v}$ are bounded by 2 , we conclude that $\sup _{p}\left|\beta_{p}^{v}\right| \leq$ $\sup _{q}\left|\beta_{2 q-1}^{v}\right|+2$ and so $\mu^{v}\left\{t: \sup _{p}\left|\beta_{p}^{v}\right|>n+2\right\} \leq 4 / n^{2}$ whenever $v \in I_{2}$. Since $\mu \leq 2 \mu^{v}$, 
the Lebesgue measure of these sets is at most $8 / n^{2}$. The same estimate holds also for $v \in-I_{2}$, since $\beta_{p}^{-v}=\beta_{p}^{v}$. Hence, denoting

$$
B=\left\{t: \sup _{p}\left|\sum_{q=0}^{p}(-1)^{q} \frac{\left\langle\beta_{q}, v^{\perp}\right\rangle}{\left\langle\beta_{q}, v\right\rangle}\right|>n+2 \text { for some } v \in W \cap \pm I_{2}\right\},
$$

we have

$$
\mu(B) \leq \frac{40}{n^{2}}
$$

We show that

$$
\mu\left(\gamma^{-1}\left(H_{n} \backslash G_{n}\right) \backslash\left(B \cup \bigcup_{p=n}^{\infty} D_{p}\right)\right)=0
$$

By (2.20) and (2.26), this will give $\mu\left(\gamma^{-1}\left(H_{n} \backslash G_{n}\right)\right)<2^{-n+1}+40 / n^{2}$, and so finish the proof.

To establish (2.27), suppose that $t \in(0,1) \backslash\left(B \cup \bigcup_{p=n}^{\infty} D_{p}\right)$ is such that $z=\gamma(t) \in H_{n} \backslash$ $G_{n}$ and all $h_{j}$ are differentiable at $z$ and simplify the notation by denoting $m_{k}(z)=m_{k}$, $w_{k}(z)=w_{k}$, and $k_{p}(z)=k_{p}$. We will need an estimate, for any $k<l$, of

$$
\left\|h_{l}^{\prime}(z)-h_{k}^{\prime}(z)\right\|=\left\|\sum_{j=k+1}^{l} 2^{-m_{j-1}} \sigma_{j-1}(z) \zeta_{j-1}(z) \varphi_{j}^{\prime}(z)\right\| .
$$

Let $p$ be the least index such that $k_{p}>k$ and let $q$ be the largest index such that $k_{q} \leq l$. Recall that $\left|\sigma_{j-1}(z)\right|=1,\left|\zeta_{j-1}(z)\right| \leq 2,\left\|\varphi_{j}^{\prime}(z)\right\| \leq 1$, and $m_{j-1} \geq m_{k}$ for all $k+1 \leq j \leq l$. Hence, the norm of each term of the series is trivially estimated by $2^{-m_{k}+1}$. If $z \notin B\left(L_{j}, \varrho_{j}\right)$, we also have $\left\|\varphi_{j}^{\prime}(z)\right\| \leq 2^{-j}$, and so the contribution of the terms for which $z \notin B\left(L_{j}, \varrho_{j}\right)$ is at most

$$
\sum_{j=k+1}^{l} 2^{-m_{k}}\left|\zeta_{j-1}(z)\right|\left\|\varphi_{j}^{\prime}(z)\right\| \leq 2^{-m_{k}} \sum_{j=k+1}^{l} 2^{-j+1} \leq 2^{-m_{k}+1}
$$

Using this, the trivial estimate for $j=k_{p}$ and $j=k_{q}$, the simple fact that $\sigma_{k_{s}-1}(z)=$ $(-1)^{s-1}$, and noting that the untreated indices $j$ are of the form $j=k_{s}$, where $p<s<q$, we get

$$
\begin{aligned}
\left\|h_{l}^{\prime}(z)-h_{k}^{\prime}(z)\right\| & \leq 6 \cdot 2^{-m_{k}}+\left\|\sum_{p<s<q} 2^{-m_{k_{s}-1}}(-1)^{s-1} \zeta_{k_{s}-1}(z) \varphi_{k_{s}}^{\prime}(z)\right\| \\
& \leq 2^{-m_{k}+3}+\left\|\sum_{p<s<q} 2^{-m_{k_{s}-1}}(-1)^{s-1} \zeta_{k_{s}-1}(z) \varphi_{k_{s}}^{\prime}(z)\right\| .
\end{aligned}
$$

A simple corollary of this is that $m_{k_{r}} \leq r$ for all $r$. Indeed, since $\left\|h_{k_{r}}^{\prime}(z)\right\| \leq C_{m_{k_{r}}}$ for all $r$ by (2.12), we get from (2.30) with $k=k_{r}$ and $l \leq k_{r+1}$ that $\left\|h_{l}^{\prime}(z)\right\| \leq C_{m_{k_{r}}}+2^{-m_{k_{r}}+3} \leq C_{m_{k_{r}}+1}$ 
for all $k_{r}<l \leq k_{r+1}$. By the definition of $m_{l}$, this gives $m_{l} \leq m_{k_{r}}+1$ for all $k_{r}<l \leq k_{r+1}$; in particular, $m_{k_{r+1}} \leq m_{k_{r}}+1$. Since this holds for all $r, m_{k_{r}} \leq r$.

We now turn our attention to the estimate of the sum in (2.30) under the special assumptions that for all $p<s<q, w_{k_{s}}=w_{k_{p}}$ and $m_{k_{s}}=m_{k_{p}} \geq n$. Since $k_{p} \geq m_{k_{p}} \geq n$, Claim 2.1 shows that $\varphi_{k_{s}}^{\prime}(z)=e_{k_{s}}^{\perp}$ and $\zeta_{k_{s}-1}(z)=1 /\left\langle e_{k_{s}}, w_{k_{p}}\right\rangle$. Hence, we wish to estimate the norm of the vector

$$
\begin{aligned}
u=u_{p, q} & :=\sum_{p<s<q} 2^{-m_{k_{s}-1}}(-1)^{s-1} \zeta_{k_{s}-1}(z) \varphi_{k_{s}}^{\prime}(z) \\
& =\sum_{p<s<q} 2^{-m_{k_{p}}}(-1)^{s-1} \frac{e_{k_{s}}^{\perp}}{\left\langle e_{k_{s}}, w_{k_{p}}\right\rangle} .
\end{aligned}
$$

Since $\left|\left\langle u^{\perp}, w_{k_{p}}\right\rangle\right|=\left|\sum_{p<s<q}(-1)^{s-1} 2^{-m_{k_{p}}}\right| \leq 2^{-m_{k_{p}}} \leq 2^{-n}$, we will establish this by estimating $\left|\left\langle u^{\perp}, w_{k_{p}}^{\perp}\right\rangle\right|$. For this, we switch from $e_{k_{s}}$ to $\beta_{s}(t)$; recall that by Claim 2.1, $e_{k_{s}} \in \pm I_{1}$, $w_{k_{p}} \in \pm I_{2}, \gamma^{\prime}(t) \in I \subset I_{1}$, therefore $\left|\left\langle e_{k_{s}}, w_{k_{p}}\right\rangle\right| \geq 1 / 2,\left|\left\langle\beta_{s}(t), w_{k_{p}}\right\rangle\right|=\left|\mathbb{E}\left(\left\langle\gamma^{\prime}, w_{k_{p}}\right\rangle \mid \Sigma_{s}\right)\right| \geq$ $1 / 2,\left\|\beta_{s}(t)\right\| \geq 1 / 2$, and $\beta_{s}(t) /\left\|\beta_{s}(t)\right\| \in I_{1}$. We also have $\left|\left\langle\beta_{s}(t), e_{k_{s}}^{\perp}\right\rangle\right| \leq 2^{-s}$ since $s>p \geq$ $m_{k_{p}} \geq n$ and so $t \notin D_{s}$, and $k_{s}(\gamma(t))<\infty$. Hence,

$$
\left|\frac{\left\langle\beta_{s}(t), w_{k_{p}}^{\perp}\right\rangle}{\left\langle\beta_{s}(t), w_{k_{p}}\right\rangle}-\frac{\left\langle e_{k_{s}}, w_{k_{p}}^{\perp}\right\rangle}{\left\langle e_{k_{s}}, w_{k_{p}}\right\rangle}\right|=\left|\frac{\left\langle\beta_{s}(t), e_{k_{s}}^{\perp}\right\rangle}{\left\langle e_{k_{s}}, w_{k_{p}}\right\rangle\left\langle\beta_{s}(t), w_{k_{p}}\right\rangle}\right| \leq 2^{-s+2},
$$

and we see from $t \notin B$ that

$$
\begin{aligned}
\left|\left\langle u^{\perp}, w_{k_{p}}^{\perp}\right\rangle\right| & \leq 2^{-m_{k_{p}}}\left(\left|\sum_{p<s<q}(-1)^{s-1} \frac{\left\langle\beta_{s}(t), w_{k_{p}}^{\perp}\right\rangle}{\left\langle\beta_{s}(t), w_{k_{p}}\right\rangle}\right|+\sum_{p<s<q} 2^{-s+2}\right) \\
& \leq 2^{-m_{k_{p}}}\left(2(n+2)+2^{-p+2}\right) \\
& \leq 2^{-n}(2 n+6) .
\end{aligned}
$$

Consequently,

$$
\left\|u_{p, q}\right\| \leq 2^{-n}(2 n+7)
$$

After this digression, we are ready to finish the argument. Since $m_{0}=0, m_{j+1} \leq m_{j}+1$, and $\sup _{j} m_{j} \geq n+2$, there are indices $j_{0}$ and $j_{1}$ such that $m_{j_{0}-1}=n, m_{j}=n+1$, for $j_{0} \leq$ $j<j_{1}$, and $m_{j_{1}}=n+2$. Let $r_{0}$ and $r_{1}$ be the least indices such that $k_{r_{0}} \geq j_{0}$ and $k_{r_{1}} \geq j_{1}$. We note that $k_{r_{0}} \geq m_{k_{r_{0}}} \geq m_{j_{0}-1}=n$. Hence, Claim 2.1 implies that there is $r_{2} \geq r_{0}$ so that $w_{k}(z)=w_{k_{r_{0}}}(z)$ for $k_{r_{0}} \leq k<k_{r_{2}}$, and $w_{k}(z)=w_{k_{r_{2}}}(z)$ for $k \geq k_{r_{2}}$. Let $r_{3}=\min \left(r_{1}, r_{2}\right)$. It follows that (2.34) can be used with $p=r_{0}$ and $q=r_{3}$ as well as with $p=r_{3}$ and $q=r_{1}$, and we get

$$
\left\|h_{j_{1}}^{\prime}(z)-h_{j_{0}-1}^{\prime}(z)\right\| \leq 2^{-n+3}+\left\|u_{r_{0}, r_{3}}\right\|+2^{-n+1}+\left\|u_{r_{3}, r_{1}}\right\| \leq 2^{-n}(4 n+24) .
$$

Since $m_{j_{0}-1}=n,\left\|h_{j_{0}-1}^{\prime}(z)\right\| \leq C_{n}$ and so

$$
\left\|h_{j_{1}}^{\prime}(z)\right\| \leq C_{n}+2^{-n}(4 n+24) \leq C_{n+1}=C_{m_{j_{1}-1}} .
$$


But this means that $n+2=m_{j_{1}}=m_{j_{1}-1}=n+1$, which is the contradiction we desired to prove (2.27), finishing the proof of the theorem.

\section{Acknowledgments}

The first author was supported by the Royal Society Wolfson Research Merit Award, and the third author was supported by the Grant GAČR 201/04/0090 and MSM 6840770010.

\section{References}

[1] G. Alberti, M. Csörnyei, and D. Preiss, Geometric structure of small sets in finite dimension and differentiability of Lipschitz maps, in preparation.

[2] T. De Pauw and P. Huovinen, Points of $\epsilon$-differentiability of Lipschitz functions from $\mathbb{R}^{n}$ to $\mathbb{R}^{n-1}$, Bull. London Math. Soc. 34 (2002), no. 5, 539-550.

[3] W. B. Johnson, J. Lindenstrauss, D. Preiss, and G. Schechtman, Almost Fréchet differentiability of Lipschitz mappings between infinite-dimensional Banach spaces, Proc. London Math. Soc. (3) 84 (2002), no. 3, 711-746.

[4] J. Lindenstrauss and D. Preiss, Almost Fréchet differentiability of finitely many Lipschitz functions, Mathematika 43 (1996), no. 2, 393-412.

[5] - On Fréchet differentiability of Lipschitz maps between Banach spaces, Ann. of Math. (2) 157 (2003), no. 1, 257-288.

[6] D. Preiss, Differentiability of Lipschitz functions on Banach spaces, J. Funct. Anal. 91 (1990), no. 2, 312-345.

[7] Z. Zahorski, Sur l'ensemble des points de non-dérivabilité d'une fonction continue, Bull. Soc. Math. France 74 (1946), 147-178 (French).

Marianna Csörnyei: Department of Mathematics, University College London, Gower Street, London WC1E 6BT, UK

E-mail address: mari@math.ucl.ac.uk

David Preiss: Department of Mathematics, University College London, Gower Street, London WC1E 6BT, UK

E-mail address: dp@math.ucl.ac.uk

Jaroslav Tišer: Department of Mathematics, Faculty of Electrical Engineering, Technical University of Prague, 16627 Prague, Czech Republic

E-mail address: tiser@math.feld.cvut.cz 


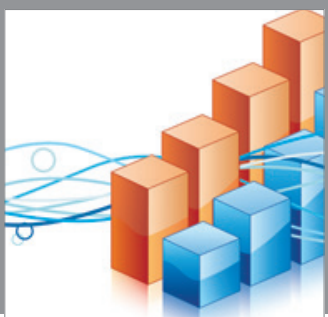

Advances in

Operations Research

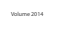

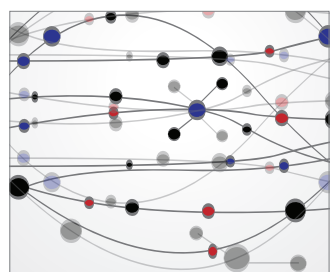

\section{The Scientific} World Journal
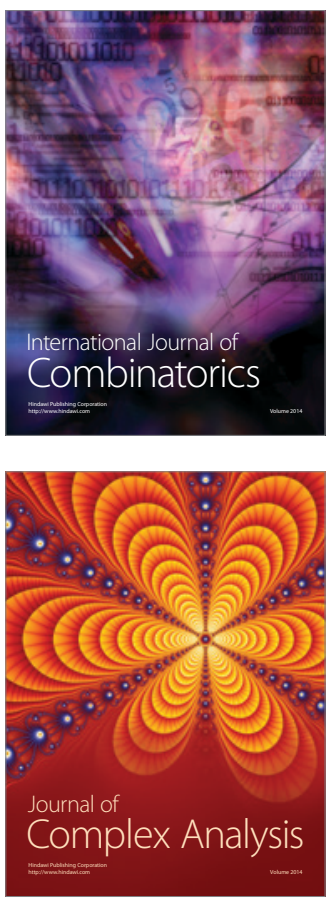

International Journal of

Mathematics and

Mathematical

Sciences
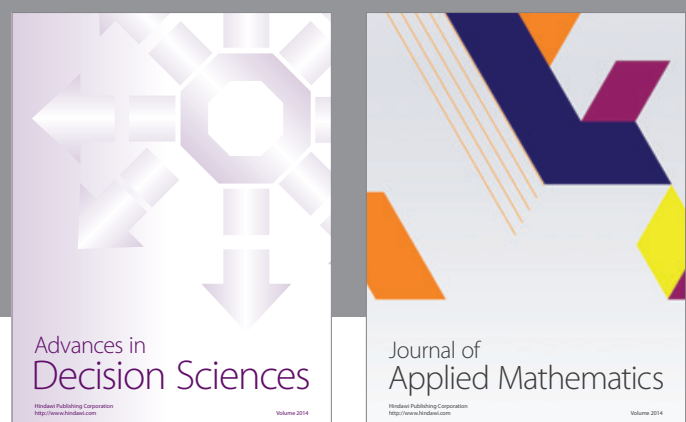

Journal of

Applied Mathematics
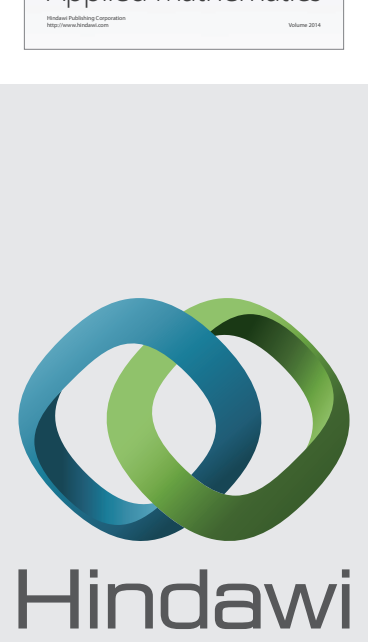

Submit your manuscripts at http://www.hindawi.com
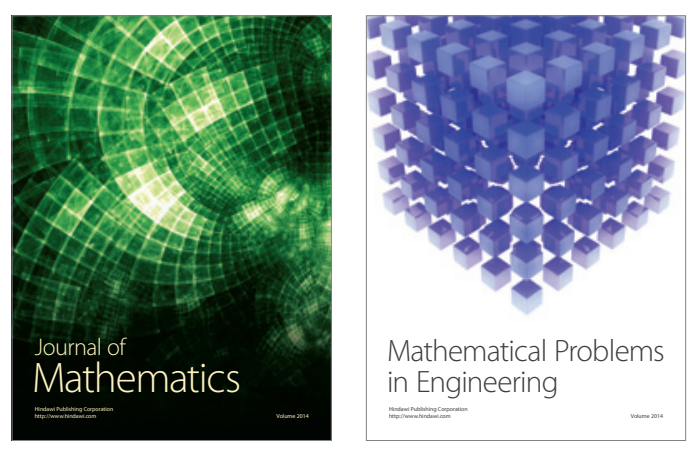

Mathematical Problems in Engineering
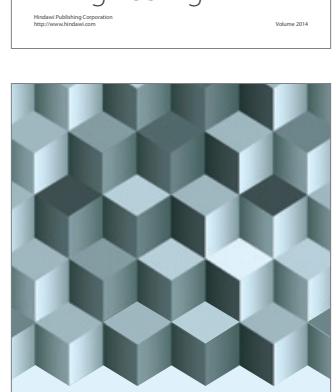

Journal of

Function Spaces
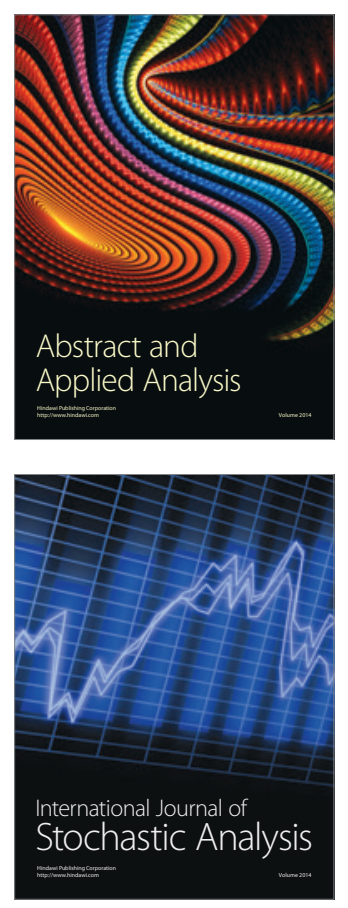

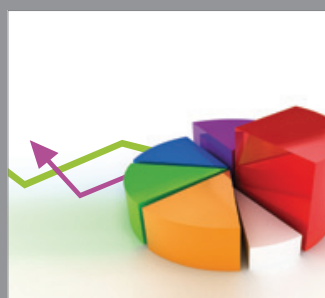

ournal of

Probability and Statistics

Promensencen
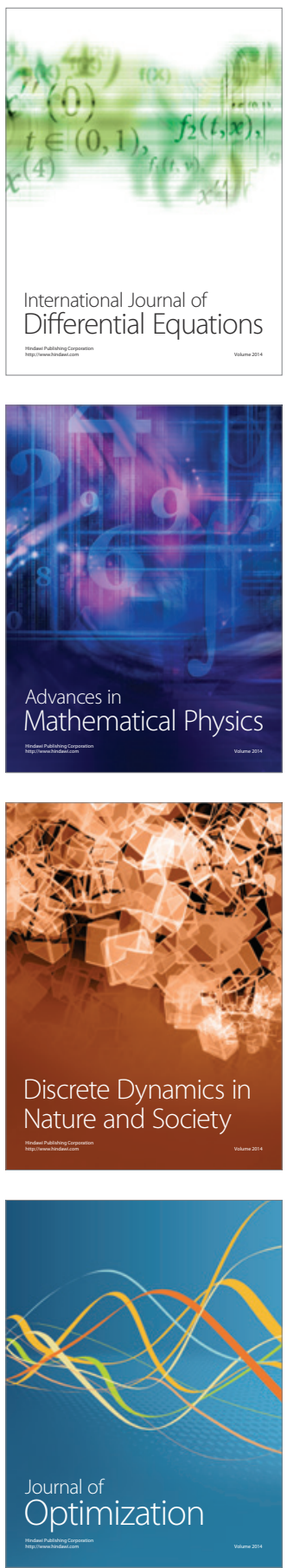